\title{
A Fully Automatic Technique for Precise Localization and Quantification of Amyloid- $\beta$ PET Scans
}

\author{
Mouna Tahmi*1, Wassim Bou-Zeid*1, and Qolamreza R. Razlighi ${ }^{1,2}$ \\ ${ }^{I}$ Department of Neurology, Columbia University Medical Center, New York, New York; and ${ }^{2}$ Department of Biomedical Engineering, \\ Columbia University, New York, New York
}

\begin{abstract}
Spatial heterogeneity in the accumulation of amyloid- $\beta$ plaques throughout the brain during asymptomatic as well as clinical stages of Alzheimer disease calls for precise localization and quantification of this protein using PET imaging. To address this need, we have developed and evaluated a technique that quantifies the extent of amyloid- $\beta$ pathology on a millimeter-by-millimeter scale in the brain with unprecedented precision using data from PET scans. Methods: An intermodal and intrasubject registration with normalized mutual information as the cost function was used to transform all FreeSurfer neuroanatomic labels into PET image space, which were subsequently used to compute regional SUV ratio (SUVR). We have evaluated our technique using postmortem histopathologic staining data from 52 older participants as the standard-of-truth measurement. Results: Our method resulted in consistently and significantly higher SUVRs in comparison to the conventional method in almost all regions of interest. A 2-way ANOVA revealed a significant main effect of method as well as a significant interaction effect of method on the relationship between computed SUVR and histopathologic staining score. Conclusion: These findings suggest that processing the amyloid- $\beta$ PET data in subjects' native space can improve the accuracy of the computed SUVRs, as they are more closely associated with the histopathologic staining data than are the results of the conventional approach.
\end{abstract}

Key Words: ${ }^{18} \mathrm{~F}$-florbetaben PET; Alzheimer disease; amyloid- $\beta$; FreeSurfer; spatial normalization

J Nucl Med 2019; 60:1771-1779

DOI: 10.2967/jnumed.119.228510

\section{$\mathbf{T}$} o quantify the aggregation of brain amyloid- $\beta$ (A $\beta)$ in vivo, different $A \beta$ tracers have been developed for PET imaging techniques, with both clinical and research applications. A $\beta$ PET scans can be evaluated qualitatively through visual reading of the tracer uptake in cortical regions by a trained radiologist (1) or quantitatively using automated or semiautomated localization methods to evaluate regional levels of tracer uptake $(2,3)$. The quantitative methods typically involve localization of tracer uptake in different regions, relative to their uptake in a reference region (ideally a region with little or no specific binding) in the PET scan; this ratio is often referred to as the regional SUV ratio

Received Mar. 19, 2019; revision accepted May 29, 2019.

For correspondence or reprints contact: Mouna Tahmi, Columbia University Medical Center, 630 W. 168th St., New York, NY 10032.

E-mail: mt3288@cumc.columbia.edu

${ }^{*}$ Contributed equally to this work.

Published online Jun. 6, 2019.

COPYRIGHT (C) 2019 by the Society of Nuclear Medicine and Molecular Imaging.
(SUVR) (4-6). Therefore, any inaccuracies in the regional delineation of the PET scan will result in a direct quantification error of the computed tracer uptake. Specifically, such inaccuracies could have detrimental effects on longitudinal $\mathrm{A} \beta$ imaging studies, which aim to track small changes in tracer uptake over time $(7,8)$. Furthermore, recent studies have demonstrated significant spatial heterogeneity in the regional pattern of $A \beta$ deposition in the brain and its spatial relationship with other brain measures, such as cortical gray matter thickness and resting-state functional connectivity, in both healthy and clinical populations $(9,10)$. Taken together, these studies highlight the significant role and clinical importance of varying topographic levels of $A \beta$ deposition, reinforcing the need for precise localization and quantification of this protein in the brain using A $\beta$ PET scans.

Most automatic quantification methods of $A \beta$ use spatial normalization to align or warp each subject's brain to a standardized template space, presumably making any voxel or region in each subject's brain comparable across all subjects in the study (11-13). The limited accuracy of conventional spatial normalization methods has been shown in other neuroimaging modalities by our group $(14,15)$ and others $(16,17)$, particularly for older subjects, who generally have significant brain atrophy even in the absence of agerelated disease (18). For example, Supplemental Video 1 demonstrates the severity of regional variability in 30 healthy and older brains after being warped to a template space using even the state-ofthe-art spatial normalization (supplemental materials are available at http://jnm.snmjournals.org).

In the present study, our aim is to address the issues associated with inaccurate spatial normalization in automatic quantification of PET scans. By circumventing the spatial normalization step and localizing tracer uptake within each subject's brain independently, we propose a native space automatic quantification method that reduces the complexity of the between-subject warping problem to a within-subject rigid-body alignment. We used the FreeSurfer parcellation and segmentation (19) tool to accurately delineate each region of interest (ROI) in subjects' native space. Then, we transferred the FreeSurfer regional mask to PET space and obtained SUVR data in each FreeSurfer region. We evaluated the accuracy of regional tracer uptake in 52 older participants who had completed both MRI and ${ }^{18} \mathrm{~F}$-florbetaben PET scans before death and who also had postmortem assessment of $A \beta$ deposition in their brain using standardized histopathologic staining, considered to be standard of truth in this study.

\section{MATERIALS AND METHODS}

\section{Participants}

The data for the present study were collected during a phase III ${ }^{18}$ F-florbetaben PET imaging study conducted from February 2010 
through July 2013 (20), where 52 subjects out of 82 recruitments (mean \pm SD age, $79.6 \pm 9.7$; age range, 58-98 y) completed both ${ }^{18} \mathrm{~F}$-florbetaben PET and MR scans in vivo before postmortem histopathologic examination. All subjects (or their legal representatives) provided written informed consent to undergo brain MRI and PET with ${ }^{18} \mathrm{~F}$-florbetaben and to donate their brains after death to undergo postmortem examination.

\section{${ }^{18}$ F-Florbetaben Imaging Acquisition}

${ }^{18} \mathrm{~F}$-florbetaben scans (as well as both MRI scans and postmortem histopathologic staining data) were provided for this study by Piramal Imaging (now known as Life Molecular Imaging). Detailed information on the ${ }^{18} \mathrm{~F}$-florbetaben PET data acquisition can be found in a previous report describing the phase III ${ }^{18} \mathrm{~F}$-florbetaben prospective study (20). Briefly, PET scans were acquired from participants $90-110 \mathrm{~min}$ after receiving an intravenous injection of $300 \mathrm{MBq}(10 \mathrm{mCi}) \pm 20 \%$ of ${ }^{18} \mathrm{~F}$-florbetaben. Reconstruction used ordered-subset expectation maximization, with attenuation correction, scatter, and random correction applied to each of the four 5-min PET frames acquired.

\section{Structural MRI Acquisition Parameters}

Each subject in the study completed a T1-weighted structural MR scanning session before death using a 1.5-T magnet and without any exogenous contrast enhancement. An accompanying T1-weighted magnetization-prepared rapid gradient-echo structural whole-brain scan (repetition time/echo time, 5-11/2-6 ms; field of view, $25.6 \times$ 16-25.6 cm; matrix size, $256 \times 160-256$; voxel size, $1.0 \times 1.0 \times$ $1.2 \mathrm{~mm}$ ) was collected for localization and spatial normalization of the PET scans for each participant.

\section{Structural MR Image Processing}

Structural T1-weighted scans were reconstructed using the FreeSurfer processing pipeline (http://surfer.nmr.mgh.harvard.edu/) to parcellate cortical ROIs and to segment subcortical ROIs. Briefly, structural images were bias field-corrected, intensity-normalized, and skull-stripped using a watershed algorithm, followed by a white matter-based segmentation, defining gray matter, white matter, and pial surfaces and correcting topology (21). In total, 95 ROI masks ( $35 \times 2$ cortical, 23 subcortical, and cerebellar gray and white matter) were extracted from the structural T1-weighted scan. On the basis of the availability of the histopathologic assessment in specific regions, 7 nonoverlapping ROIs were selected to compute regional SUVRs: caudal and rostral middle frontal, caudal anterior cingulate, posterior cingulate, precuneus, hippocampus, parahippocampal gyrus, and lateral occipital cortex. Supplemental Table 1 lists the regions for which we have the histopathology data available, alongside their corresponding FreeSurfer regions.

\section{Histopathologic Staining Procedure}

Brain samples from participants who passed away during the study were used to obtain histopathologic confirmation of $A \beta$ presence and density in the brain, as previously described (20). Briefly, postmortem histopathologic examination was assessed according to the 4 histologically defined scores of the Consortium for Establishing a Registry for Alzheimer Disease (22). The onsite pathologist conducted the autopsy within $36 \mathrm{~h}$ of death and performed the scoring. Tissue was classified as 1 for absent, 2 for sparse, 3 for moderate, or 4 for frequent, depending on the neuritic plaque densities, as detected by Bielschowsky silver staining. Eight brain regions were dissected, including the middle frontal gyrus, anterior cingulate cortex, posterior cingulate cortex, precuneus, hippocampus, parahippocampal gyrus, occipital cortex, and whole cerebellum.

\section{Native Space Method of Quantification}

A flowchart of the steps in the proposed native space method has been illustrated in Figure 1.
The first step in the native space method is to create a static PET image by aligning the 4 dynamic PET frames to the first frame using rigid-body registration to correct for potential head motion during the imaging session (six degrees of freedom [DoF], correlation) as seen in Figure 1A. Subsequently, these 4 registered frames are averaged together (voxelwise operation) to create a single static PET image (Fig. 1B). In addition, our proposed method takes advantage of the existing CT scan (often acquired for attenuation correction in PET) to further improve the accuracy of intermodal registration, since the information on the subject's skull is completely lost in PET scans but is visible in CT scans. The static PET and CT scans are coregistered using a rigid-body intermodal registration (six DoF, normalized mutual information) and merged to generate a composite image in PET static space (Fig. 1C).

Each participant's structural T1-weighted scan in FreeSurfer space is also registered to the participant's merged image using rigid-body registration (six DoF, normalized mutual information) to obtain the second transformation matrix. A combination of the 2 transformation matrices is used to transfer the regional masks (ROIs) (including the cerebellum) from FreeSurfer space to static PET image space. The SUVR map was generated by normalizing each voxel's uptake to the averaged uptake in a reference region, which in our study was the whole cerebellum. For the purposes of the present study, SUVRs were calculated within the 7 aforementioned ROIs in which the histopathologic examination was also performed.

\section{Standard Space Quantification}

To compare our proposed native space quantification method to the commonly used conventional method in the field, we also computed the SUVR measurements in the 7 selected ROIs using a standard space method that uses the Montreal Neurological Institute (MNI) template and the automated anatomic labeling (AAL) atlas (23). In the standard space method, we first generated a static PET image from 4 dynamic scans as described in the native space method. We then registered each participant's static PET scan to that subject's T1-weighted MRI scan using rigid-body transformation (six DoF, correlation) (Fig. 2B). Tissue segmentation was also performed on each subject's MRI scan to obtain the whole-brain gray-matter tissue mask (Fig. 2C). Next, the T1-weighted structural MRI scan was registered to the MNI template using a state-of-the-art nonlinear registration technique called advanced normalization tools (Fig. 2B) (24,25). The combination of the transformations and warping field was then applied to the PET image, generating a spatially normalized PET image in MNI template space, as well as a spatially normalized whole-brain gray matter mask. The intersection of the ROI masks in the AAL atlas with the whole-brain gray matter mask generated the regional mask, which was then used to compute regional SUVRs by dividing the average regional uptake within each ROI by that of the whole cerebellum, as obtained directly from the AAL atlas. In addition, to evaluate our method using matching ROIs, we have also repeated the standard space technique using the FreeSurfergenerated ROIs in the MNI template space.

\section{Overall Quality Assessment of Registration}

Both registration and segmentation algorithms are susceptible to large misalignments. In some cases, even the orientation of the images can be mismatched. To make sure our results were not driven by these bulk misalignments, we visually inspected the quality of the registration and segmentation in both native space and standard space. We found no major misalignment in native or standard space methods.

\section{Statistical Analysis}

All statistical analysis in this work was performed using Microsoft Excel and the JMP 14.0 statistical software package for Microsoft Office. A 2-way ANOVA including all subjects was conducted to test 


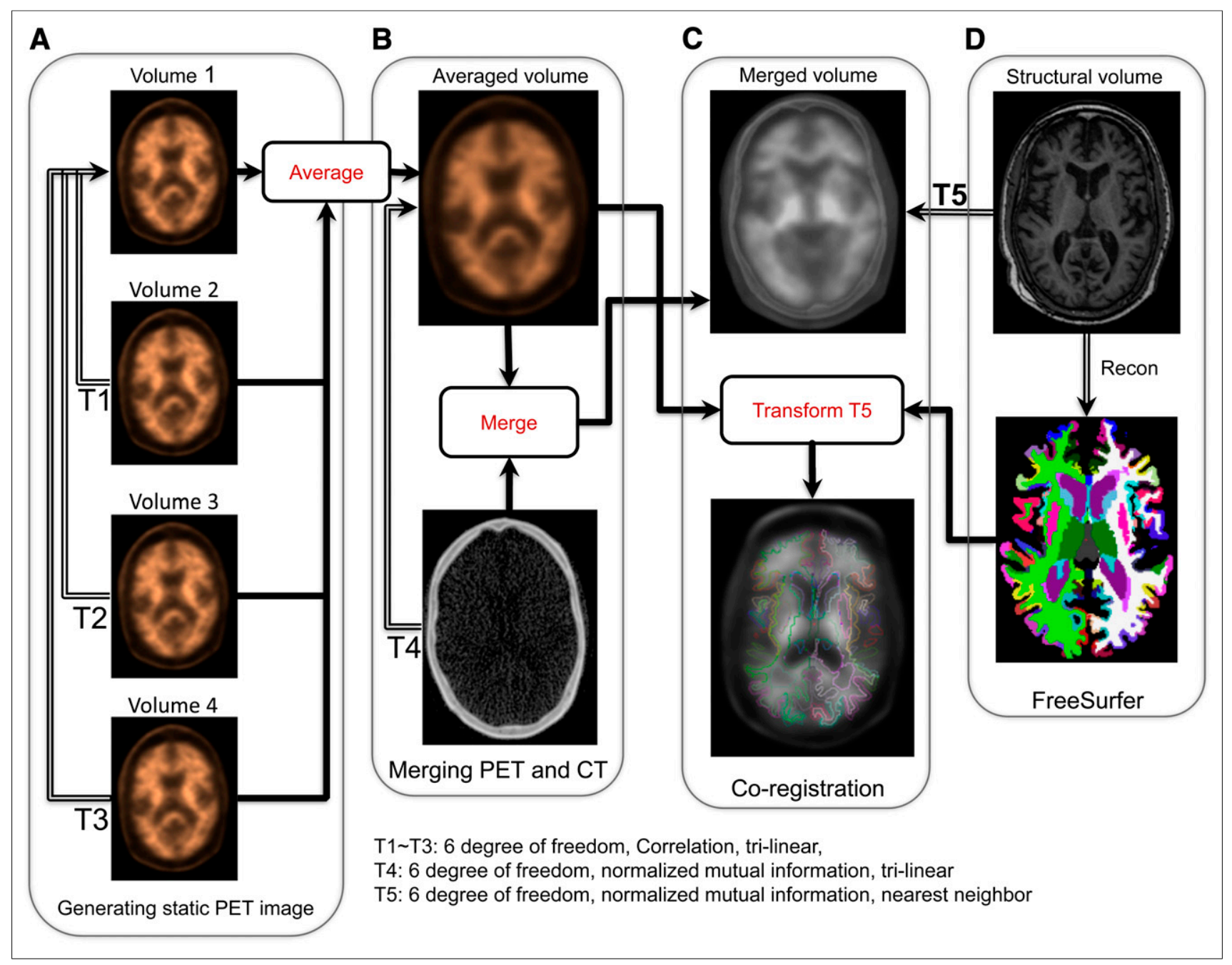

FIGURE 1. Flowchart of our PET image reconstruction technique (native space): generation of static PET image (A), merging of PET and CT (B), coregistration (C), and FreeSurfer segmentation (D). T1 T5 denote the 5 different transformation matrices obtained by rigid-body registrations.

the effect of the 2 independent variables (method: native space vs. standard space; histopathologic staining scores: 1 for absent, 2 for sparse, 3 for moderate, or 4 for frequent) and their interaction on the SUVR measurements. This analysis was performed both for aggregated data (by combining data from all 7 available regions) and for each region separately. Post hoc Student $t$ tests were performed to further investigate the effect of the normalization method at each level of the histopathologic staining. Differences in slope tests were used as secondary post hoc tests to examine the interaction effects on every region and on the aggregated data. Higher slopes should indicate a stronger correlation, or predictive power, between the measured SUVRs and histopathologic staining scores. Finally, standard $F$ tests were used to assess any difference in SUVR variance obtained from the two methods.

\section{RESULTS}

Table 1 summarizes the demographics of the 52 participants who were scanned with ${ }^{18} \mathrm{~F}$-florbetaben and underwent postmortem histopathologic evaluation for $A \beta$ burden. Of the 52 participants, $33(63.5 \%)$ were classified as probable Alzheimer disease, $12(23 \%)$ as other dementia patients, and $7(13.5 \%)$ as nondemented volunteers. There were no significant differences among the 3 groups in age or sex $\left(t_{\text {age }}<1.7, P_{\text {age }}>0.07 ; t_{\text {sex }}<1.34, P_{\text {sex }}>0.2\right)$.
A 2-way ANOVA (factors: method and histopathologic score) performed on the aggregated data showed a main effect of method $\left(F_{(1,706)}=198.80, P<0.0001\right)$, a main effect of histopathologic staining score $\left(F_{(3,706)}=87.73, P<0.0001\right)$, and a significant method $\times$ histopathology interaction $\left(F_{(3,706)}=6.43, P=0.0114\right)$. We then explored these significant findings further: first, by investigating the main effect of method. Table 2 lists the results of Student $t$ tests for two independent SUVR measurements, indicating whether the two methods generated significantly different SUVRs for each histopathologic staining score within each region. As evident from these results, the mean SUVRs were significantly higher for the native space method than for the standard method

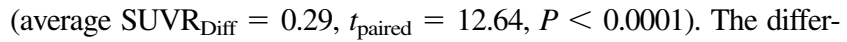
ence in the mean SUVRs were also significant for all 7 selected brain regions and every corresponding histopathologic level $(t>2.5, P<$ 0.05), except the middle frontal level $3(t=1.93 P=0.06)$ and the occipital level $2(t=1.99 P=0.056)$, which were only marginally significant. Figure 3 also illustrates these differences in mean SUVR using box plots. Overall, the precuneus, posterior cingulate, and anterior cingulate showed higher SUVRs than the rest of the regions.

Next, to investigate the interaction between method and histopathologic score, we conducted a difference-in-slope test between 


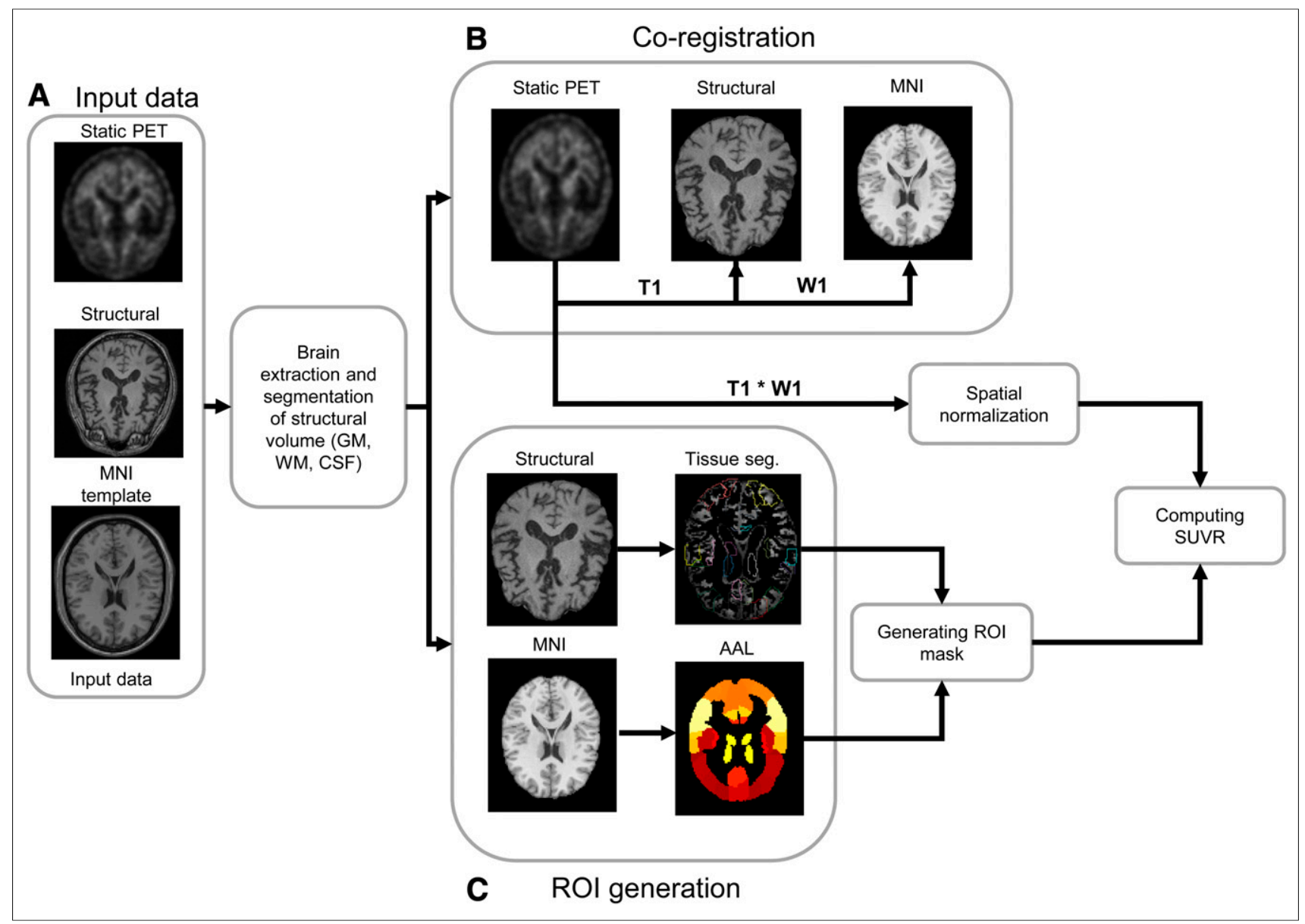

FIGURE 2. Flowchart of standard PET image reconstruction technique (standard space method): input data (A), coregistration (B), and ROI generation (C). $\mathrm{CSF}=$ cerebrospinal fluid; $\mathrm{GM}=$ gray matter; $\mathrm{WM}=$ white matter. $\mathrm{T} 1$ denotes the transformation matrices obtained by rigid-body registration, and $\mathrm{W} 1$ denotes the warping field obtained by nonlinear registration.

the 2 methods in the aggregated dataset, as well as in the 7 preselected ROIs. As seen in Figure 4, the native space method improved the slope of the relationship between histopathologic score and SUVR measurement significantly compared with the standard space method $\left(\beta_{\mathrm{diff}}=0.05, P<0.01\right)$ in the aggregated data. We then looked at this effect in the different regions separately. As seen in Figure 3, slopes, in general, were higher with the native space method than with the standard method in all 7 ROIs. Native space results showed a significant positive slope in 6 of the 7 selected

TABLE 1

Demographics of Scanned and Autopsied Research Subjects

\begin{tabular}{cccc}
\hline Parameter & $\begin{array}{c}\text { Alzheimer } \\
\text { disease } \\
(n=33)\end{array}$ & $\begin{array}{c}\text { Other } \\
\text { dementias } \\
(n=12)\end{array}$ & $\begin{array}{c}\text { Nondemented } \\
\text { volunteers } \\
(n=7)\end{array}$ \\
\hline $\begin{array}{c}\text { Mean age } \\
\pm \text { SD }(y)\end{array}$ & $78.76 \pm 10.09$ & $79.67 \pm 8.28$ & $87.14 \pm 9.84$ \\
Sex $(n)$ & 20 & 3 & 9 \\
M & 13 & 4 & 3 \\
\hline F & & & \\
\hline
\end{tabular}

ROIs $(\beta>0.049, P<0.009)$ with 1 nonsignificant slope in the hippocampus $(\beta=0.02, P=0.25)$. On the other hand, using the standard space method, only 4 of 7 ROIs showed a significant positive slope ( $\beta>0.039, P<0.012$ ). Furthermore, the regional slope difference tests between the two methods were significant in the precuneus $\left(\beta_{\mathrm{diff}}=0.1, P=0.028\right)$ and posterior cingulate gyrus ( $\beta_{\text {diff }}=0.121, P=0.017$ ), whereas the slope difference tests for the rest of the regions did not reach significance (Fig. 3).

We also performed $F$ tests to assess any significant differences in variance associated with each histopathologic stage in each region for both methods (Supplemental Table. 2). The results of the $F$ tests revealed no significant differences in any regions and their corresponding histopathologic levels, except for 4 cases in which the native space method resulted in either lower variance (hippocampus stage $4 ; F_{(8,8)}=0.23, P=0.02$ ) or higher variance (posterior cingulate stage 2, occipital stages 1 and $3 ; F_{(12,12)}>3.13$, $P<0.03$ ). In addition, repeating the standard space method using the FreeSurfer regions in MNI space instead of the AAL atlas did not improve its results. Supplemental Figure 1 illustrates the results of this analysis, where the native space method outperformed the standard space method in 4 of 7 ROIs (precuneus, posterior cingulate, anterior cingulate, and middle frontal). Since the results of native space have not been altered, this indicates that the standard space results with 
TABLE 2

SUVRs for Native and Standard Methods Based on Pathology Levels and $t$ Test Results Comparing 2 Methods

\begin{tabular}{|c|c|c|c|c|}
\hline $\mathrm{ROI}$ & Histologic score & Native SUVR & Standard SUVR & $P$ \\
\hline \multirow[t]{4}{*}{ Middle frontal } & $1(n=12)$ & $0.77 \pm 0.12$ & $0.62 \pm 0.17$ & $<0.05^{\star}$ \\
\hline & $2(n=17)$ & $1.11 \pm 0.32$ & $0.83 \pm 0.24$ & $<0.01^{*}$ \\
\hline & $3(n=13)$ & $1.22 \pm 0.29$ & $1.03 \pm 0.20$ & 0.06 \\
\hline & $4(n=10)$ & $1.27 \pm 0.26$ & $0.93 \pm 0.19$ & $<0.01^{\star}$ \\
\hline \multirow[t]{4}{*}{ Anterior cingulate } & $1(n=14)$ & $0.86 \pm 0.18$ & $0.65 \pm 0.23$ & $0.01^{*}$ \\
\hline & $2(n=21)$ & $1.33 \pm 0.33$ & $1.01 \pm 0.30$ & $<0.01^{\star}$ \\
\hline & $3(n=10)$ & $1.34 \pm 0.26$ & $0.97 \pm 0.28$ & $<0.01^{\star}$ \\
\hline & $4(n=6)$ & $1.45 \pm 0.27$ & $1.08 \pm 0.13$ & $0.01^{*}$ \\
\hline \multirow[t]{4}{*}{ Posterior cingulate } & $1(n=16)$ & $1.02 \pm 0.22$ & $0.83 \pm 0.19$ & $0.01^{*}$ \\
\hline & $2(n=18)$ & $1.42 \pm 0.34$ & $1.01 \pm 0.14$ & $<0.001^{\star}$ \\
\hline & $3(n=8)$ & $1.56 \pm 0.29$ & $0.93 \pm 0.35$ & $0.001^{*}$ \\
\hline & $4(n=9)$ & $1.53 \pm 0.29$ & $1.04 \pm 0.28$ & $<0.01^{\star}$ \\
\hline \multirow[t]{4}{*}{ Precuneal } & $1(n=14)$ & $0.89 \pm 0.1$ & $0.7 \pm 0.11$ & $<0.001^{\star}$ \\
\hline & $2(n=21)$ & $1.37 \pm 0.30$ & $0.95 \pm 0.2$ & $<0.001^{\star}$ \\
\hline & $3(n=7)$ & $1.46 \pm 0.2$ & $1.00 \pm 0.24$ & $<0.01^{\star}$ \\
\hline & $4(n=9)$ & $1.49 \pm 0.27$ & $0.99 \pm 0.16$ & $<0.001^{\star}$ \\
\hline \multirow[t]{4}{*}{ Hippocampal } & $1(n=17)$ & $0.88 \pm 0.15$ & $0.63 \pm 0.19$ & $<0.001^{\star}$ \\
\hline & $2(n=14)$ & $0.95 \pm 0.15$ & $0.73 \pm 0.19$ & $<0.01^{\star}$ \\
\hline & $3(n=12)$ & $0.88 \pm 0.09$ & $0.64 \pm 0.11$ & $<0.001^{\star}$ \\
\hline & $4(n=9)$ & $0.97 \pm 0.11$ & $0.68 \pm 0.22$ & $<0.01^{\star}$ \\
\hline \multirow[t]{4}{*}{ Parahippocampal } & $1(n=17)$ & $0.81 \pm 0.1$ & $0.63 \pm 0.14$ & $<0.001^{\star}$ \\
\hline & $2(n=14)$ & $1.01 \pm 0.18$ & $0.71 \pm 0.21$ & $<0.001^{\star}$ \\
\hline & $3(n=7)$ & $0.97 \pm 0.13$ & $0.75 \pm 0.13$ & $<0.01^{\star}$ \\
\hline & $4(n=11)$ & $0.99 \pm 0.17$ & $0.72 \pm 0.13$ & $<0.001^{\star}$ \\
\hline \multirow[t]{4}{*}{ Occipital } & $1(n=13)$ & $0.82 \pm 0.12$ & $0.69 \pm 0.07$ & $<0.01^{\star}$ \\
\hline & $2(n=14)$ & $1.01 \pm 0.23$ & $0.84 \pm 0.19$ & 0.056 \\
\hline & $3(n=10)$ & $1.1 \pm 0.21$ & $0.82 \pm 0.1$ & $0.001^{*}$ \\
\hline & $4(n=14)$ & $1.04 \pm 0.22$ & $0.86 \pm 0.15$ & $0.01^{*}$ \\
\hline
\end{tabular}

*Significantly higher mean SUVR for native method than for standard method.

SUVRs are mean \pm SD.

FreeSurfer regions have a substantially decreased correlation with histopathologic score in comparison to the standard space results with AAL atlas ROIs.

\section{DISCUSSION}

The goal of this study was to introduce and evaluate our native space technique in quantifying $A \beta$ accumulation as imaged by ${ }^{18}$ F-florbetaben PET scans in comparison to the standard space approach commonly used to analyze A $\beta$ PET data $(20,26)$. Our findings suggest that the native space method using FreeSurfer's parcellation and segmentation outperforms the standard space approach based on the MNI template and AAL atlas. Considering the histopathologic assessment as the standard of truth for $A \beta$ quantification, we show that the SUVR measurements obtained through the native space method not only are significantly higher than the ones obtained through the standard space method in almost all available brain regions and every histopathologic staining level but also are significantly more predictive of the histopathologic staining scores, suggesting higher precision of $\mathrm{A} \beta$ quantification.

Recently, and in line with our work, Schwarz et al. (27), investigated the effects of imprecise rigid-body registration between A $\beta$ PET and MRI on SUVR measurement. The authors reported that imprecision in PET/MRI rigid-body registration contributes to imprecise change over time measurements by approximately $7 \%$ of the expected reference values for annual change in subjects with Alzheimer disease pathology. According to the authors, this imprecision increases with $A \beta$ load. Brain $A \beta$ aggregation, on the other hand, reflects significant spatial heterogeneity. Frontal, posterior cingulate, and precuneus regions have been widely reported as regions with the highest levels of $\mathrm{A} \beta$ deposition in Alzheimer disease brains $(6,20,28)$. More importantly, studies looking at $A \beta$ deposition in early Alzheimer disease and in mild cognitive impairment have shown that these regions are areas of initial $A \beta$ deposition, suggesting that deposition starts in these regions and continues to accrue as the disease progresses $(29,30)$. Interestingly, 


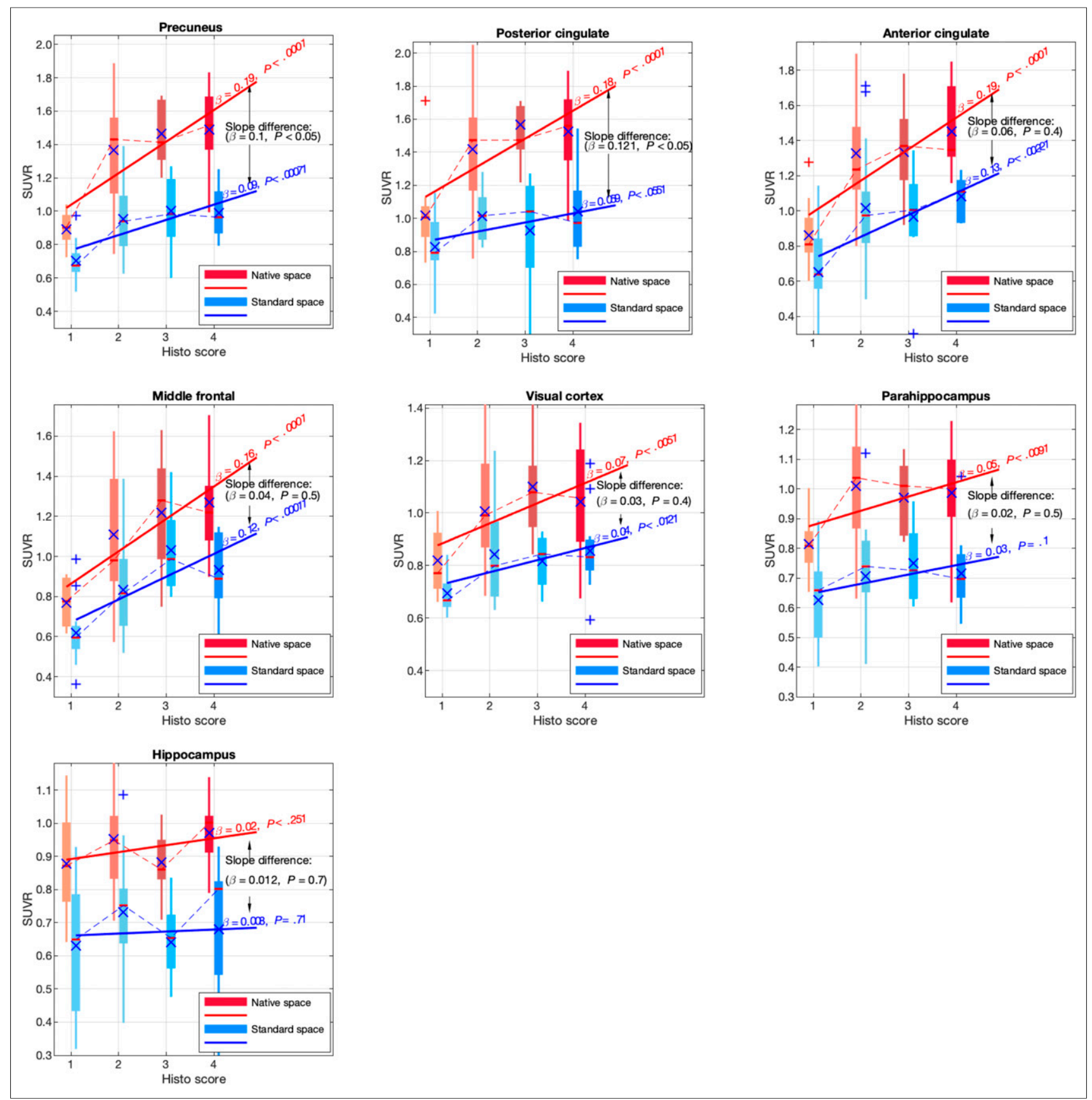

FIGURE 3. Box-and-whisker plots with slope test parameters showing SUVR distribution obtained using native and standard methods at each level of postmortem histopathologic staging. Depicted is distribution of regional uptake for each of 7 selected ROIs in separate plots. $\times$ indicates the mean, - indicates the median, and + shows the outliers.

and in light of the results of the current study, the posterior cingulate and precuneus were regions in which the native space method demonstrated higher and significant correlations with histopathology, as well as the most significant improvement in the precision of $\mathrm{A} \beta$ quantification when compared with the standard space method. Thus, the native space method could potentially enhance the ability to detect $A \beta$ in the preclinical phases of Alzheimer disease, which is of importance as there is growing interest in detecting $A \beta$ earlier in life, as well as understanding the progression of its deposition in vivo (i.e., Grothe's $A \beta$ imaging staging (31)).
The native space SUVR from the hippocampus did not show any relationship with histopathologic staging scores. This may raise concern that for quantifying SUVRs within smaller regions, the native space method might not be as effective as it is for larger regions. However, we argue that this is, in fact, not the case. First, unlike results using the conventional method, the native space SUVR for the parahippocampal region (a brain region with similar size in the medial temporal lobe) shows a significant relationship with histopathologic staging scores. Second, the hippocampal regions in the brain are shown to lack sensitivity for $\mathrm{A} \beta$ compared with the other regions included in the present study (20). 


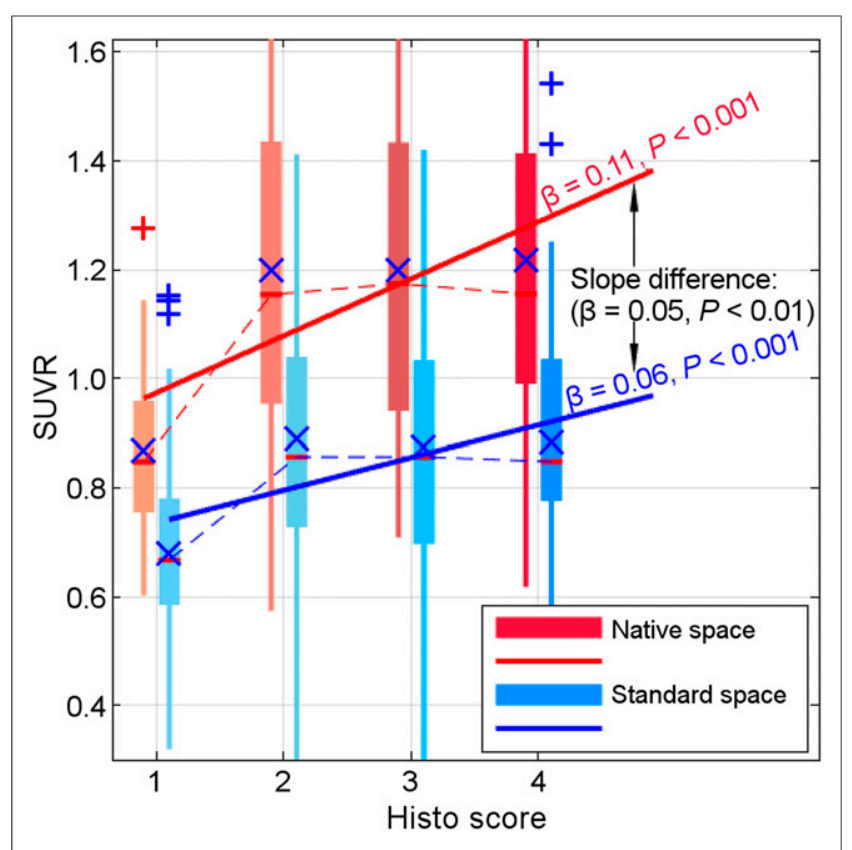

FIGURE 4. Box plots Illustrate the distribution of SUVRs computed with native and standard space method at each level of histopathologic stage from aggregated data (all regions). Lines depict the regression slope computed separately for native and standard space, indicating the strength of relationship between SUVR and histopathologic staging. $\times$ indicates the mean, - indicates the median, and + shows the outliers.

Third, the conventionally computed SUVRs from the hippocampus, as well as from the parahippocampus regions, are quite low (SUVR $<<1$ for all 4 staining scores), which indicates that $A \beta$ deposition is much lower in these regions than in the reference region (presumably with no A $\beta$ deposition), suggesting that the SUVRs computed using the standard space method are biased toward lower SUVRs. Finally, one can expect that the level of imprecision in ROI delineation (particularly in small regions) using the standard space method could have a large effect on corresponding SUVRs. For these reasons, we think that the effectiveness of the native space method is likely not altered in smaller regions and that this lack of relationship between native space computed SUVRs and histopathologic staining scores in the hippocampus is, in fact, due to the lack of significant $A \beta$ deposition.

We have used the proposed method in previously published studies in our division (32-34); however, the actual evaluation of our method using the standard of truth has not been performed previously. In our previous studies, our analysis pipeline took advantage of the existing CT scan, which has the registration advantage of showing skull bone structure. In the current study, CT data were unfortunately missing from the imaging dataset that was used; however, we still had significant improvement in SUVR measurement compared with the standard method. Hence, we are confident that if CT data were available, our results would be only more robustly in favor of the native space method.

It is noteworthy that other MRI template spaces (e.g., Talairach space) or atlases can be used in the standard space method, as shown in previous research including ${ }^{18} \mathrm{~F}$-florbetaben PET $(4,6)$. However, all standard space methods are based on spatial normalization, which requires nonlinear registration of data to a standard template. Therefore, we expect SUVRs produced using these other template methods to have a degree of imprecision similar to that of
SUVRs produced using the native space method, in which there is no need for spatial normalization. Repeating our analysis for the standard method using the FreeSurfer regions in MNI space to ensure the comparability of ROIs between native space and the standard method did not improve the results of the standard space method, and native space still outperformed the standard space with the FreeSurfer labels in 4 of 7 ROIs (Supplemental Fig. 1). We think there are two reasons that the standard space method with FreeSurfer labels did not improve the results. First, the cortical regions in the FreeSurfer atlas from MNI space are relatively thin; therefore, even a small misalignment or inaccuracy in spatial normalization would completely shift the ROI to white matter or cerebrospinal fluid, whereas the AAL atlas, which covers not only gray matter but also a large portion of the white matter and cerebrospinal fluid, could be less sensitive to such a small misalignment. Second, in our native space method, we use a gray matter mask obtained by a wholebrain tissue segmentation tool, as done in previous studies $(26,35)$, to exclude binding in white matter and cerebrospinal fluid. We did not compare our method with MR-free quantification methods that are based on PET-to-PET registration to a common template $(2,36)$, because MRI is largely acquired in PET quantification studies, as well as in clinical trials of Alzheimer disease. Only large-scale studies evaluating all other template approaches (MR-based or MR-free), using in vivo $\mathrm{A} \beta$ PET alongside postmortem histopathologic assessment, will be able to assess the complete effect of all methods of spatial normalization on A $\beta$ PET data validity.

Our method was applied only to A $\beta$ PET data obtained using the ${ }^{18} \mathrm{~F}$-florbetaben tracer. We did not test this method using other $\mathrm{A} \beta$ PET tracers, such as ${ }^{11} \mathrm{C}$-Pittsburgh compound $\mathrm{B}$, or other ${ }^{18} \mathrm{~F}$ labeled tracers $\left({ }^{18} \mathrm{~F}\right.$-florbetapir and ${ }^{18} \mathrm{~F}$-flutemetamol). These tracers also use SUVR quantification, typically computed through a process that involves registration of the PET scan to a standard space using spatial normalization. Thus, whereas the present study includes only PET data obtained using the ${ }^{18} \mathrm{~F}$-florbetaben tracer, it is expected that spatial normalization would significantly affect the accuracy of PET data obtained using any of the available A $\beta$ PET tracers. However, future studies should formally assess whether the standard space method has similar effects on data from all amyloid PET tracers.

Nowadays, it is common practice to collect neuroimaging data from multiple centers for large clinical studies. Because reducing between-center variability continues to be an issue for multicenter studies, it would be beneficial to assess whether use of the native space method would mitigate or exacerbate multicenter variability or noise. Using the center information in the Piramal phase III dataset to conduct a 1-way ANOVA of the effect of center on SUVRs, we have detected significant center-related variability in the standard space SUVRs acquired from different sites $\left(F_{(9,353)}=\right.$ $2.12, P<0.01)$, which was significantly attenuated using the native space method $\left(F_{(9,353)}=1.18, P=0.33\right)$. Together, these findings suggest that using the native space method could potentially reduce the variability due to acquiring data from different sites in the computed SUVRs, which could also be beneficial in processing multicenter PET data. However, further investigation using a much larger sample is required to properly investigate this effect in the future. Furthermore, future research must assess the impact of the native space method on SUVR measurement precision in other datasets, such as the ${ }^{18} \mathrm{~F}$-florbetaben test-retest scans (37), which would be critical for use of amyloid PET as a treatment endpoint in antiamyloid clinical trials. Additionally, to directly compare results across studies, another future study should test the effect or 
benefit of the native space method, in comparison to a spatially normalized method, in the ${ }^{18} \mathrm{~F}$-florbetaben centiloid datasets (38).

Finally, we are aware of the fact that the degree of precision of SUVR measurement in A $\beta$ PET imaging can also be affected by other factors such as the choice of the reference region, the type of $A \beta$ tracer, the scan duration relative to injection timing, the size of ROIs, and the use of partial-volume correction, all of which have been widely addressed in previous studies $(8,39,40)$. In this work, our automatic quantification method specifically focuses on the effect of an understudied SUVR measurement factor, spatial normalization, which subsequently affects the precision of ROI localization. Luckily, spatial normalization is a postprocessing step that can easily be eliminated, as is the case using our native space approach, and can even be applied to existing A $\beta$ PET data, which therefore could improve the precision of SUVR measurement in past studies retrospectively.

\section{CONCLUSION}

We have highlighted the inaccuracy of the regional SUVR measurements obtained from the quantification of $A \beta$ PET scans with ${ }^{18} \mathrm{~F}$-florbetaben tracer using the conventional standard space technique. We proposed a more accurate quantification method by circumventing the problematic nonlinear registration step and processing the PET scans in subjects' native space. We evaluated our proposed method by showing that native space regional SUVRs are more closely related to standardized histopathologic staging scores than the regional SUVRs computed using the conventional standard space technique. This approach could especially benefit studies targeting early detection and regional differentiation of brain amyloid burden, which is important especially in view of the regional heterogeneity in brain $A \beta$ deposition.

\section{DISCLOSURE}

This work was funded by NIH-NIA grant R01 AG057962 and a Taub Institute MRI pilot platform seed award. No other potential conflict of interest relevant to this article was reported.

\section{ACKNOWLEDGMENTS}

Special thanks are given to Andrew Stephens, Susan De Santi, and Santiago Bullich (Life Molecular Imaging, formerly known as Piramal Imaging) and to Osama Sabri (Department of Nuclear Medicine, University of Leipzig) as the principal investigator of the phase III study for providing MRI, PET, and histopathologic scoring data; Hengda He for preparing box plot figures; and Eleanna Varangis for proofreading the manuscript.

\section{KEY POINTS}

QUESTION: Does analyzing brain A $\beta$ PET imaging in subjects' native space (without spatial normalization) improve the accuracy of the resulting SUVRs compared with the same analysis in standard space (with spatial normalization)?

PERTINENT FINDINGS: Using our native space method resulted in significantly increased measurement precision of $A \beta$ compared with a commonly used standard method that relies on spatial normalization.

IMPLICATIONS FOR PATIENT CARE: Our method can be applied to existing or future $A \beta$ PET data to improve the accuracy of SUVRs, especially when tracking small changes in deposition over the course of Alzheimer pathology.

\section{REFERENCES}

1. Seibyl J, Catafau AM, Barthel H, et al. Impact of training method on the robustness of the visual assessment of ${ }^{18} \mathrm{~F}$-florbetaben PET scans: results from a phase3 study. J Nucl Med. 2016;57:900-906.

2. Joshi AD, Pontecorvo MJ, Lu M, Skovronsky DM, Mintun MA, Devous MDS. A semiautomated method for quantification of F 18 florbetapir PET images. J Nucl Med. 2015;56:1736-1741.

3. Zubal G, Wisniewski G, Seibyl J. Automated software package for analyzing new beta-amyloid radioligands in Alzheimer's patients [abstract]. J Nucl Med. 2008;49(suppl):378P-378P.

4. Fleisher AS. Using positron emission tomography and florbetapir F 18 to image cortical amyloid in patients with mild cognitive impairment or dementia due to Alzheimer disease. Arch Neurol. 2011;68:1404-1411.

5. Jack CR, Lowe VJ, Senjem ML, et al. ${ }^{11} \mathrm{C} \mathrm{PiB}$ and structural MRI provide complementary information in imaging of Alzheimer's disease and amnestic mild cognitive impairment. Brain. 2008;131:665-680.

6. Villemagne VL, Ong K, Mulligan RS, et al. Amyloid imaging with ${ }^{18} \mathrm{~F}-$ florbetaben in Alzheimer disease and other dementias. J Nucl Med. 2011;52: 1210-1217.

7. Landau SM, Breault C, Joshi AD, et al. Amyloid- $\beta$ imaging with Pittsburgh compound $\mathrm{B}$ and florbetapir: comparing radiotracers and quantification methods. J Nucl Med. 2013;54:70-77.

8. Schmidt ME, Chiao P, Klein G, et al. The influence of biological and technical factors on quantitative analysis of amyloid PET: points to consider and recommendations for controlling variability in longitudinal data. Alzheimers Dement. 2015;11:1050-1068.

9. Mormino EC, Smiljic A, Hayenga AO, et al. Relationships between beta-amyloid and functional connectivity in different components of the default mode network in aging. Cereb Cortex. 2011;21:2399-2407.

10. Doherty BM, Schultz SA, Oh JM, et al. Amyloid burden, cortical thickness, and cognitive function in the Wisconsin Registry for Alzheimer's Prevention. Alzheimers Dement (Amst). 2015;1:160-169.

11. Talairach J, Tournoux P. Co-Planar Stereotaxic Atlas of the Human Brain: 3Dimensional Proportional System - An Approach To Cerebral Imaging. New York, NY: Thieme Medical Publishers; 1988.

12. Mazziotta J, Toga A, Evans A, et al. A probabilistic atlas and reference system for the human brain: International Consortium for Brain Mapping (ICBM). Philos Trans R Soc Lond B Biol Sci. 2001;356:1293-1322.

13. Camus V, Payoux P, Barré L, et al. Using PET with ${ }^{18} \mathrm{~F}-\mathrm{AV}-45$ (florbetapir) to quantify brain amyloid load in a clinical environment. Eur J Nucl Med Mol Imaging. 2012;39:621-631.

14. Razlighi QR, Habeck C, Steffener J, et al. Unilateral disruptions in the default network with aging in native space. Brain Behav. 2014;4:143-157.

15. Liu X, Gerraty RT, Grinband J, Parker D, Razlighi QR. Brain atrophy can introduce age-related differences in BOLD response. Hum Brain Mapp. 2017;38: 3402-3414.

16. Seibert TM, Brewer JB. Default network correlations analyzed on native surfaces. J Neurosci Methods. 2011;198:301-311.

17. Senjem ML, Gunter JL, Shiung MM, Petersen RC, Jack CRJ. Comparison of different methodological implementations of voxel-based morphometry in neurodegenerative disease. Neuroimage. 2005;26:600-608.

18. Fjell AM, Walhovd KB, Fennema-Notestine C, et al. One-year brain atrophy evident in healthy aging. J Neurosci. 2009;29:15223-15231.

19. Fischl B, van der Kouwe A, Destrieux C, et al. Automatically parcellating the human cerebral cortex. Cereb Cortex. 2004;14:11-22.

20. Sabri O, Sabbagh MN, Seibyl J, et al. Florbetaben PET imaging to detect amyloid beta plaques in Alzheimer's disease: phase 3 study. Alzheimers Dement. 2015;11:964-974.

21. Dale AM, Fischl B, Sereno MI. Cortical surface-based analysis. Neuroimage. 1999;9:179-194.

22. Mirra SS, Heyman A, McKeel D, et al. The Consortium to Establish a Registry for Alzheimer's Disease (CERAD). Part II. Standardization of the neuropathologic assessment of Alzheimer's disease. Neurology. 1991;41:479-486.

23. Tzourio-Mazoyer N, Landeau B, Papathanassiou D, et al. Automated anatomical labeling of activations in SPM using a macroscopic anatomical parcellation of the MNI MRI single-subject brain. Neuroimage. 2002;15:273289.

24. Klein A, Ghosh SS, Avants B, et al. Evaluation of volume-based and surfacebased brain image registration methods. Neuroimage. 2010;51:214-220.

25. Avants BB, Epstein CL, Grossman M, Gee JC. Symmetric diffeomorphic image registration with cross-correlation: evaluating automated labeling of elderly and neurodegenerative brain. Med Image Anal. 2008;12:26-41. 
26. Barthel H, Gertz H-J, Dresel S, et al. Cerebral amyloid- $\beta$ PET with florbetaben $\left({ }^{18} \mathrm{~F}\right)$ in patients with Alzheimer's disease and healthy controls: a multicentre phase 2 diagnostic study. Lancet Neurol. 2011;10:424-435.

27. Schwarz CG, Jones DT, Gunter JL, et al. Contributions of imprecision in PETMRI rigid registration to imprecision in amyloid PET SUVR measurements. Hum Brain Mapp. 2017;38:3323-3336.

28. Hsiao IT, Huang CC, Hsieh CJ, et al. Perfusion-like template and standardized normalization-based brain image analysis using ${ }^{18} \mathrm{~F}$-florbetapir (AV-45/Amyvid) PET. Eur J Nucl Med Mol Imaging. 2013;40:908-920.

29. Braak H, Braak E. Neuropathological stageing of Alzheimer-related changes. Acta Neuropathol (Berl). 1991;82:239-259.

30. Fodero-Tavoletti MT, Rowe CC, McLean CA, et al. Characterization of PiB binding to white matter in Alzheimer disease and other dementias. J Nucl Med. 2009;50: 198-204.

31. Grothe MJ, Barthel H, Sepulcre J, Dyrba M, Sabri O, Teipel SJ. In vivo staging of regional amyloid deposition. Neurology. 2017;89:2031-2038.

32. Oh H, Steffener J, Razlighi QR, et al. A $\beta$-related hyperactivation in frontoparietal control regions in cognitively normal elderly. Neurobiol Aging. 2015;36:3247-3254.

33. Oh H, Steffener J, Razlighi QR, Habeck C, Stern Y. Beta-amyloid deposition is associated with decreased right prefrontal activation during task switching among cognitively normal elderly. J Neurosci. 2016;36:1962-1970.
34. Gu Y, Razlighi QR, Zahodne LB, et al. Brain amyloid deposition and longitudinal cognitive decline in nondemented older subjects: results from a multi-ethnic population. PLoS One. 2015;10:e0123743.

35. Lilja J, Leuzy A, Chiotis K, Savitcheva I, Sörensen J, Nordberg A. Spatial normalization of 18 F-flutemetamol PET images using an adaptive principalcomponent template. J Nucl Med. 2018;60:285-291.

36. Raniga P, Bourgeat P, Ourselin S, Villemagne V, O'Keefe G, Rowe C. PIB-PET segmentation for automatic SUVR normalisation without MR information. 4th IEEE International Symposium on Biomedical Imaging: From Nano to Macro. Piscataway, NJ: IEEE; 2007:348-351.

37. Sabri O, Seibyl J, Rowe C, Barthel H. Beta-amyloid imaging with florbetaben. Clin Transl Imaging. 2015;3:13-26.

38. Rowe CC, Doré V, Jones G, et al. ${ }^{18} \mathrm{~F}$-florbetaben PET beta-amyloid binding expressed in Centiloids. Eur J Nucl Med Mol Imaging. 2017;44:2053-2059.

39. Carbonell F, Zijdenbos AP, Charil A, Grand'Maison M, Bedell BJ. Optimal target region for subject classification on the basis of amyloid PET images. J Nucl Med. 2015;56:1351-1358.

40. Landau SM, Fero A, Baker SL, et al. Measurement of longitudinal beta-amyloid change with ${ }^{18} \mathrm{~F}$-florbetapir PET and standardized uptake value ratios. J Nucl Med. 2015;56:567-574. 